\title{
Nomogram for Predicting Survival for Oral Squamous Cell Carcinoma
}

\author{
Ki-Yeol Kim ${ }^{1}$, ShengJin $\mathrm{Li}^{1}$ and In-Ho Cha ${ }^{1,2 *}$ \\ ${ }^{1}$ Oral Cancer Research Institute, College of Dentistry, \\ Yonsei University, Seoul 120-752, Korea, ${ }^{2}$ Department \\ of Oral and Maxillofacial Surgery, College of Dentistry, \\ Yonsei University, Seoul 120-752, Korea
}

\begin{abstract}
An accurate system for predicting the survival of patients with oral squamous cell carcinoma (OSCC) will be useful for selecting appropriate therapies. A nomogram for predicting survival was constructed from 96 patients with primary OSCC who underwent surgical resection between January 1994 and June 2003 at the Yonsei Dental Hospital in Seoul, Korea. We performed univariate and multivariate Cox regression to identify survival prognostic factors. For the early stage patients group, the nomogram was able to predict the 5 and 10 year survival from OSCC with a concordance index of 0.72 . The total point assigned by the nomogram was a significant factor for predicting survival. This nomogram was able to accurately predict the survival after treatment of an individual patient with OSCC and may have practical utility for deciding adjuvant treatment.
\end{abstract}

Keywords: c-index, nomogram, oral squamoue cell carcinoma, predictive model, survival

\section{Introduction}

Oral cancer is the sixth most common cancer in men and the twelfth most common cancer in women (Sudbo and Reith, 2005). According to statistics from 1999 to 2002 , the annual incidence rate of oral cancer is $5.9 \%$ in men and $2.2 \%$ in women in South Korea (based on data from the National Cancer Center in Korea). The approximately $90 \%$ of oral is squamous cell carcinoma (Pisani et al., 1999). Patients with oral squamous cell carcinoma (OSCC) are often asymptomatic, but may experience minimal pain in the early stage (Epstein et al., 2007). Despite the availability of advanced clinical diagnostic systems, including computed tomography (CT),

*Corresponding author: E-mail cha8764@yuhs.ac

Tel + 82-2-2228-3140, Fax + 82-2-392-2959

Accepted 25 November 2010 magnetic resonance imaging (MRI), positron emission tomography/computed tomography (PET/CT) imaging and improved therapy, the overall 5-year survival rates of OSCC over the past two decades are still poor (Myoung et al., 2006). Therefore, it is necessary to develop novel prognostic tools for predicting the OSCC patients' status, which could improve survival by allowing enough time for the appropriate therapy to be implemented.

Stephenson et al. (2005) showed that the prediction accuracy was improved by the integration of clinical variables and gene expression (Stephenson et al., 2005b). It has already been shown that the expression level of specific genes influence survival. Specific protein expression was used to predict survival in various cancers including clear cell renal carcinoma (Kim et al., 2004). The relationship between p53 and the survival in OSCC has been studied by many researchers (Galli et al., 2009; Goulart et al., 2009; Shah et al., 2009; Smith et al., 2009). In these studies, some reported that p53 predicted the survival of OSCC (Galli et al., 2009; Shah et al., 2009) while others did not (Goulart et al., 2009; Smith et al., 2009), and Smith et al. (2009) concluded that p53 does not predict the progression of OSCC after an extensive review of the previous studies.

In this study, significant clinical factors for predicting survival were identified and a nomogram was constructed using these factors. In addition, we evaluated the effect of p53 on the survival of OSCC patients, by embedding it into the nomogram. The created nomogram was evaluated by c-index.

Nomogram is a graphical representation of a statistical model and provides the probability of a particular clinical outcome, such as death or recurrence (Kattan et al., 1998). It has been constructed for predicting survival in prostate cancer (Kattan et al., 1999; Stephenson et al., 2005a), vulvar cancer (Rouzier et al., 2006), osteosarcoma (Kim et al., 2009), renal cancer (Karakiewicz et al., 2007), breast cancer (Rouzier et al., 2005), and advanced Non-Small-Cell Lung cancer (Hoang et al., 2005). Furthermore, nomograms have been shown to be superior to the traditional staging systems in predicting the features of various cancers (Hoang et al., 2005; Karakiewicz et al., 2007; Kattan et al., 1999; Kim et al., 2009; Rouzier et al., 2006; Rouzier et al., 2005; Stephenson et al., 2005a). 


\section{Methods}

\section{Patients}

We retrospectively investigated 96 patients $(71$ men and 25 women) with primary OSCC who underwent surgical ablation between January 1994 and June 2003 at the Yonsei Dental Hospital in Seoul, Korea.

The tumor location was the tongue in 26 cases, gingiva in 56 cases, and other sites in 14 cases, which included the buccal cheek, floor of mouth and lower lip. The pathological stage was divided into two groups, early and advanced. The I and II stages were grouped into the early group, and the III and IV stages were grouped into the advanced group. The pathologic (pTMN) classification and staging were classified according to the $6^{\text {th }}$ edition of AJCC (American Joint Committee on Cancer).

\section{Immunohistochemical staining}

Immunohistochemical staining for p53 was performed using an EnVision-HRP detection system (Dako, Carpinteria, CA, USA). The primary antibody was the p53 monoclonal antibody, which specifically stained p53 (Dako). Sections (3 $\mu \mathrm{m}$ thick) were cut from tumor tissue blocks mounted on slides and sections were dried for $1-2$ hours at $56^{\circ} \mathrm{C}$. Briefly, sections were deparaffinized in xylene and were rehydrated in graded alcohol. After antigen retrieval by the addition of citrate buffer $(\mathrm{pH}, 6.0)$ with the use of an autoclave at full power for $4 \mathrm{~min}$, tissue sections were treated with $3 \%$ hydrogen peroxide for $10 \mathrm{~min}$ to block endogenous peroxidase. Sections were incubated with p53 (1:50) in a humid chamber overnight at $4^{\circ} \mathrm{C}$.

Slides were then incubated with Envision reagent followed by incubation with Diaminobenzidine (DAB) chromogen, slides were counterstained with Mayer's hematoxylin, and then mounted. All of the stained sections were imaged by a light microscope.

\section{Immunohistochemical analysis}

Immunohistochemical staining was evaluated by an investigator who did not know the clinicopathological characteristics or the clinical outcome of the patients. Specimens were considered positive for staining when the tumor cells had dark brown nuclei, and specimens were considered negative for staining when the tumor cells had only blue nuclei. The percentage of stained tumor cells was graded using a four-point scale $(0,+1$, $+2,+3,+4)$ as follows: 0 , none of the tumor cells were stained; $+1, \leq 25 \%$ of the tumor cells were stained; $+2,25 \%$ to $\leq 50 \%$ of the tumor cells were stained; $+3,50 \%$ to $\leq 75 \%$ of the tumor cells were stained; $+4,>75 \%$ of tumor cells were stained. The intensity was divided into negative, weak, moderate and strong $(0,+1,+2,+3)$.

\section{Histological grading}

OSCC tissue specimens were subjected to routine hematoxylin and eosin ( $\mathrm{H}$ \& E) staining. Specimens were graded into well (G1), moderately (G2) and poorly (G3) differentiated squamous cell carcinomas using the World Health Organization (WHO) grading system. Thirty-one cases were well differentiated, 49 cases were moderately differentiated, and 16 cases were poorly differentiated.

\section{Statistical analysis}

The relationships between the clinical factors were tested using the Chi-square statistic. Univariate and multivariate analyses were performed to assess the effects of various factors on the prediction of survival. The Cox proportional hazard model was used to perform the multivariate analysis.

Nomogram for the prediction of survival of OSCC patients was created with the selected significant variables and evaluated by the concordance index (c-index). Calibration was assessed by plotting the predicted versus the actual probability. All statistical analyses were performed using $\mathrm{R}$ with the Design, eha and Hmisc libraries.

\section{Results and Discussion}

The clinical features and p53 expression of the 96 patients used in this study are summarized in Table 1. They underwent surgical resection between January 1994 and June 2003 at the Severance Hospital in Seoul, Korea. The association of clinical factors and pathological groups was shown using $p$-values from chi-square test in Table 1.

Tumor site, LN metastasis and T stage were significantly and differentially distributed between the early and advanced groups. The outcome pattern was also significantly different. This result indicated that early and advanced groups have different clinical characteristics; therefore, the predictive model should be identified for each stratified groups. In regards to tumor sites, $32.5 \%$ of gingiva was in the early stage while $77 \%$ was in the advanced group. Lymph node metastasis was not observed in the early group while over $60 \%$ was in the ad- 
Table 1. Characteristics of patients with OSCC $(\mathrm{N}=96)$

\begin{tabular}{|c|c|c|c|c|}
\hline Clinical variables & $\begin{array}{c}\text { Patients } \\
(\mathrm{n}=96)\end{array}$ & $\begin{array}{l}\text { Early stage } \\
\qquad(\mathrm{n}=40)\end{array}$ & $\begin{array}{l}\text { Advanced stage } \\
\quad(n=56)\end{array}$ & $\begin{array}{c}\mathrm{p} \text {-value } \\
\text { (Early vs. Advanced) }\end{array}$ \\
\hline \multicolumn{5}{|l|}{ Age } \\
\hline$<60$ & 42 (56\%) & $16(40 \%)$ & $26(46 \%)$ & \\
\hline$\geq 60$ & $54(44 \%)$ & $24(60 \%)$ & $30(54 \%)$ & 0.6764 \\
\hline \multicolumn{5}{|l|}{ Sex } \\
\hline Female & $25(26 \%)$ & $9(22.5 \%)$ & $16(29 \%)$ & \\
\hline Male & $71(74 \%)$ & $31(77.5 \%)$ & $40(71 \%)$ & 0.6654 \\
\hline \multicolumn{5}{|l|}{ LN metastasis $(n=73)$} \\
\hline$N(-)$ & $62(65 \%)$ & $40(100 \%)$ & 22 (39\%) & \\
\hline $\mathrm{N}(+)$ & $34(35 \%)$ & $0(0 \%)$ & $34(61 \%)$ & $3.304 \mathrm{e}-09$ \\
\hline \multicolumn{5}{|l|}{ Tumor site } \\
\hline Gingiva & $56(58 \%)$ & $13(32,5 \%)$ & $43(77 \%)$ & \\
\hline Tongue & $26(27 \%)$ & $18(40 \%)$ & $8(14 \%)$ & \\
\hline Other & $14(25 \%)$ & $9(22.5 \%)$ & $5(9 \%)$ & $7.793 \mathrm{e}-05$ \\
\hline \multicolumn{5}{|l|}{ Histopathologic grade } \\
\hline G1 & $31(32 \%)$ & $13(32.5 \%)$ & $18(32 \%)$ & \\
\hline G2 & $49(51 \%)$ & 22 (55\%) & 27 (48\%) & \\
\hline G3 & $16(17 \%)$ & $5(12.5 \%)$ & $11(20 \%)$ & 0.6295 \\
\hline \multicolumn{5}{|l|}{ Distant metastasis } \\
\hline No & $89(92 \%)$ & 37 (92.5\%) & 52 (93\%) & \\
\hline Yes & 7 (8\%) & $3(7.5 \%)$ & $4(7 \%)$ & 0.74 \\
\hline \multicolumn{5}{|c|}{ Pathologic stage ( $P$ stage $)$} \\
\hline Early & $40(42 \%)$ & $40(100 \%)$ & $0(0 \%)$ & \\
\hline Advanced & $56(58 \%)$ & $0(0 \%)$ & $56(100 \%)$ & NA \\
\hline \multicolumn{5}{|l|}{ T stage } \\
\hline $\mathrm{T} 1$ & $19(20 \%)$ & $16(40 \%)$ & $3(5.5 \%)$ & \\
\hline T2 & $33(34 \%)$ & $24(60 \%)$ & $9(16 \%)$ & \\
\hline T3 & $23(24 \%)$ & $0(0 \%)$ & $23(41 \%)$ & \\
\hline $\mathrm{T} 4$ & $21(22 \%)$ & $0(0 \%)$ & $21(37.5 \%)$ & $1.127 \mathrm{e}-12$ \\
\hline \multicolumn{5}{|l|}{ p53 } \\
\hline-+ & $37(39 \%)$ & $26(60 \%)$ & $32(57 \%)$ & \\
\hline+++++ & $59(61 \%)$ & $14(40 \%)$ & $24(43 \%)$ & 0.3755 \\
\hline \multicolumn{5}{|l|}{ Disease outcome } \\
\hline Survived & $45(47 \%)$ & $24(60 \%)$ & $21(37.5 \%)$ & \\
\hline Dead & $51(53 \%)$ & $16(40 \%)$ & $35(62.5 \%)$ & 0.04878 \\
\hline
\end{tabular}

vanced group.

The primary end point was survival time. The free-survival interval was defined as the time between the date of surgery to the last visit or the date when the patient died. Univariate and multivariate analyses were carried out to identify the characteristics associated with survival. Table 2 shows the results of the univariate and multivariate Cox regression analysis for the whole dataset.

$P$ stage and LN metastasis were significant factors in the univariate analysis and no significant factors were identified in the multivariate analysis. Therefore, it would be desirable to construct a predictive model separately using the $P$ stage. It would also be possible to construct separate models using LN metastasis, because $\mathrm{LN}$ metastasis was shown to be a significant factor in the univariate analysis.

To explore the association of p53 expression with other clinical factors, a chi-square test was performed. The results were summarized in Table 3 .

From Table 3, p53 was shown to not be significantly associated with any clinical factors in the whole dataset and advanced group, and it was only significantly associated with the Tumor Site in the early group. These results indicated that p53 can be a novel and independent predictor in the prediction model. In the separate analysis of the early and advanced groups, LN metastasis had only one category, 'negative' , in the early group; therefore, we excluded this variable from the model (Table 4).

No significant factors were identified in the advanced group. However, Grade was significant and p53 was shown to be slightly significant in the early group, even though the p-value was only marginally larger than 0.05 . Therefore, we constructed a nomogram for the early stage of OSCC. 
Table 2. Univariate and multivariate analysis using the whole dataset $(n=96)$

\begin{tabular}{|c|c|c|c|c|}
\hline \multirow{2}{*}{ Variables } & \multicolumn{2}{|c|}{ Univariate } & \multicolumn{2}{|c|}{ Multivariate } \\
\hline & HR (95\% Cl) & $p$ & $\mathrm{HR}(95 \% \mathrm{Cl})$ & $\mathrm{p}$ \\
\hline \multicolumn{5}{|l|}{ LN metastasis } \\
\hline \multicolumn{5}{|l|}{ Histopathologic Grade } \\
\hline G1: G2 & $0.84(0.44,1.59)$ & 0.591 & $0.82(0.41,1.64)$ & 0.566 \\
\hline G3 : G2 & $1.23(0.58,2.60)$ & 0.350 & $1.66(0.74,3.74)$ & 0.133 \\
\hline \multicolumn{5}{|l|}{ Distant Metastasis } \\
\hline$N: Y$ & $0.44(0.11,1.80)$ & 0.250 & $0.32(0.07,1.57)$ & 0.160 \\
\hline \multicolumn{5}{|l|}{$P$ stage } \\
\hline early : advanced & $0.54(0.3,0.98)$ & 0.044 & $0.89(0.20,3.26)$ & 0.861 \\
\hline \multicolumn{5}{|l|}{ Tumor site } \\
\hline Tongue : gingiva & $0.72(0.36,1.44)$ & 0.359 & $1.09(0.47,2.53)$ & 0.837 \\
\hline Other : gingiva & $1.14(0.54,2.40)$ & 0.732 & $1.99(0.79,5.03)$ & 0.145 \\
\hline \multicolumn{5}{|l|}{$\mathrm{T}$ stage } \\
\hline T1 : T2 & $0.62(0.25,1.57)$ & 0.3147 & $0.66(0.25,1.74)$ & 0.401 \\
\hline Т3: Т2 & $1.09(0.53,2.25)$ & 0.2655 & $1.53(0.48,4.88)$ & 0.243 \\
\hline T4 : T2 & $1.77(0.88,3.56)$ & 0.1333 & $2.08(0.73,5.89)$ & 0.098 \\
\hline \multicolumn{5}{|l|}{ p53 } \\
\hline$-+:+++++$ & $1.26(0.73,2.20)$ & 0.409 & $1.58(0.84,2.99)$ & 0.150 \\
\hline
\end{tabular}

Table 3. Relationship between p53 \& clinical characteristics

\begin{tabular}{|c|c|c|c|c|c|}
\hline \multirow[b]{2}{*}{ Variables } & \multicolumn{2}{|c|}{ p53 } & \multicolumn{3}{|c|}{$\mathrm{p}$-value } \\
\hline & $-\quad+(n=37)$ & $++\quad+++(n=59)$ & $\begin{array}{l}\text { Whole data set } \\
\qquad(\mathrm{n}=96)\end{array}$ & Early $(n=40)$ & Advanced $(n=56)$ \\
\hline \multicolumn{6}{|l|}{ LN matastasus $(\mathrm{N}=73)$} \\
\hline $\mathrm{n}(-)$ & $27(73 \%)$ & $35(59 \%)$ & 0.2535 & NA & 0.5495 \\
\hline$n(+)$ & $10(27 \%)$ & $24(41 \%)$ & & & \\
\hline \multicolumn{6}{|l|}{ Tumor site } \\
\hline Gingiva & $23(62 \%)$ & 33 (56\%) & & & \\
\hline Tongue & $10(27 \%)$ & $16(27 \%)$ & 0.6911 & 0.01633 & 0.4086 \\
\hline Other & $4(11 \%)$ & $10(17 \%)$ & & & \\
\hline \multicolumn{6}{|l|}{ Histopathologic grade } \\
\hline G1 & $15(41 \%)$ & $16(27 \%)$ & & & \\
\hline G2 & $17(46 \%)$ & $32(54 \%)$ & 0.3802 & 0.1794 & 0.4652 \\
\hline G3 & $5(13 \%)$ & $11(19 \%)$ & & & \\
\hline \multicolumn{6}{|l|}{ Distant metastasis } \\
\hline No & 35 (95\%) & $54(92 \%)$ & 0.8732 & 0.3051 & 0.8756 \\
\hline Yes & $2(5 \%)$ & $5(8 \%)$ & & & \\
\hline \multicolumn{6}{|l|}{$P$ stage } \\
\hline Early & $18(49 \%)$ & $19(32 \%)$ & 0.3755 & NA & NA \\
\hline Advanced & $19(51 \%)$ & 37 (68\%) & & & \\
\hline \multicolumn{6}{|l|}{ T stage } \\
\hline $\mathrm{T} 1$ & 7 (19\%) & $12(20 \%)$ & & & \\
\hline T2 & $15(41 \%)$ & $18(31 \%)$ & 0.6654 & 0.8457 & 0.4697 \\
\hline Т3 & $9(24 \%)$ & $14(24 \%)$ & & & \\
\hline T4 & $6(16 \%)$ & $15(25 \%)$ & & & \\
\hline
\end{tabular}

A nomogram predicting the 5-year and 10-year survival with clinical factors and p53 expression was constructed for the early group (Fig. 1). Metastasis was excluded from the model identification because it was highly unbalanced between the two categories ( $\mathrm{Y}$ and N).

The C-index was 0.72 in the nomogram for the early group and p53 was not an influential factor in prediction 
Table 4. Multivariate Cox regression for early stage $(n=40)$ and advanced stage $(n=56)$

\begin{tabular}{|c|c|c|c|c|}
\hline \multirow{2}{*}{ Variables } & \multicolumn{2}{|c|}{ Early stage } & \multicolumn{2}{|c|}{ Advanced stage } \\
\hline & $\mathrm{HR}(95 \% \mathrm{Cl})$ & $\mathrm{p}$ & $\mathrm{HR}(95 \% \mathrm{Cl})$ & $p$ \\
\hline \multicolumn{5}{|l|}{ LN metastasis } \\
\hline$-:+$ & NA & NA & $0.55(0.21,1.42)$ & 0.2139 \\
\hline \multicolumn{5}{|l|}{ Histopathologic grade } \\
\hline G1: G2 & $0.24(0.06,0.94)$ & 0.0392 & $1.72(0.73,4.03)$ & 0.2135 \\
\hline G3 : G2 & $6.56(1.22,35.40)$ & 0.0016 & $1.37(0.50,3.71)$ & 0.6838 \\
\hline \multicolumn{5}{|l|}{ Distant merastasis } \\
\hline $\mathrm{Y}: \mathrm{N}$ & $0.51(0.05,5.52)$ & 0.57808 & $0.38(0.05,3.14)$ & 0.3694 \\
\hline \multicolumn{5}{|l|}{ Tumor site } \\
\hline Tongue : gingiva & $1.20(0.33,4.33)$ & 0.7825 & $1.30(0.42,4.08)$ & 0.6486 \\
\hline Other : gingiva & $3.32(0.76,14.59)$ & 0.1589 & $3.26(0.87,12.21)$ & 0.0797 \\
\hline \multicolumn{5}{|l|}{$\mathrm{T}$ stage } \\
\hline T1 : T2 & $0.41(0.11,1.54)$ & 0.18639 & & \\
\hline T1: T3 & & & $0.24(0.03,1.87)$ & 0.3005 \\
\hline T2 : T3 & & & $0.66(0.19,2.29)$ & 0.1721 \\
\hline T4: T3 & & & $144(0,57,365)$ & 0.0805 \\
\hline \multicolumn{5}{|l|}{ p53 } \\
\hline$-+:+++++$ & $266(087,811)$ & 008570 & $130(0,59,285)$ & 05174 \\
\hline
\end{tabular}

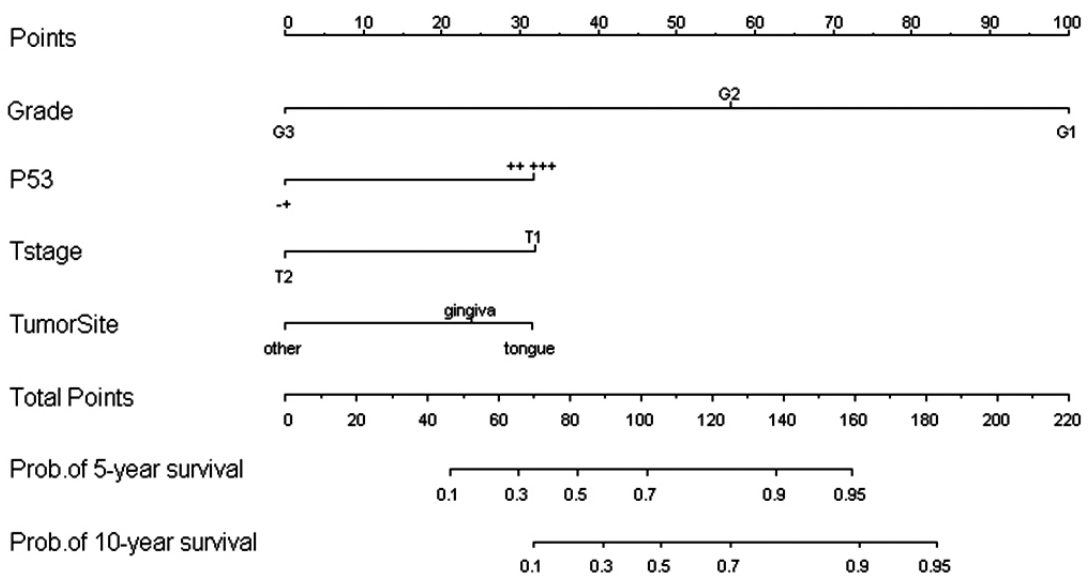

Fig. 1. Nomogram for predicting the 5-year and 10-year survival after surgery on OSCC patients. even though it was slightly significant in the multivariate analysis. The nomogram operates by summing the points of each independent covariate. The probabilities of 5-year and 10-year survival are identified by the calculated total point (Fig. 2).

Fig. 2 shows that the probability of free-survival increased as the total points increased. For example, if the total point was 140 for a patient, the probabilities of 5 and 10 year survival would be $90 \%$ and $78 \%$, respectively. We also investigated if the total point calculated by the nomogram would be a novel predictor. The mean of total points assigned by the nomogram was 116.5 and we stratified patients into two groups using this value. The sample size for the early stage was 40 , which was not sufficient to identify subsets; how- ever, we examined if the total points derived from the nomogram could significantly classify the survival of OSCC. For this, the Kaplan-Meier survival curve was plotted and the Log-Rank test was performed (Fig. 3).

Fig. 3 shows the stratification of the survival curves based on total points assigned by the nomogram, and those curves were significantly different ( $p$-value $<0.05)$. This $p$-value should be considered as a measure of curve separation. Based on the results shown in Figure 3 , the total point calculated by nomogram could be a combined marker for predicting survival.

The nomogram can be a model in which known prognostic factors can be combined and used for risk prediction in cancer (Bianco, 2006). One advantage of nomograms is that they are weighted models comprised 

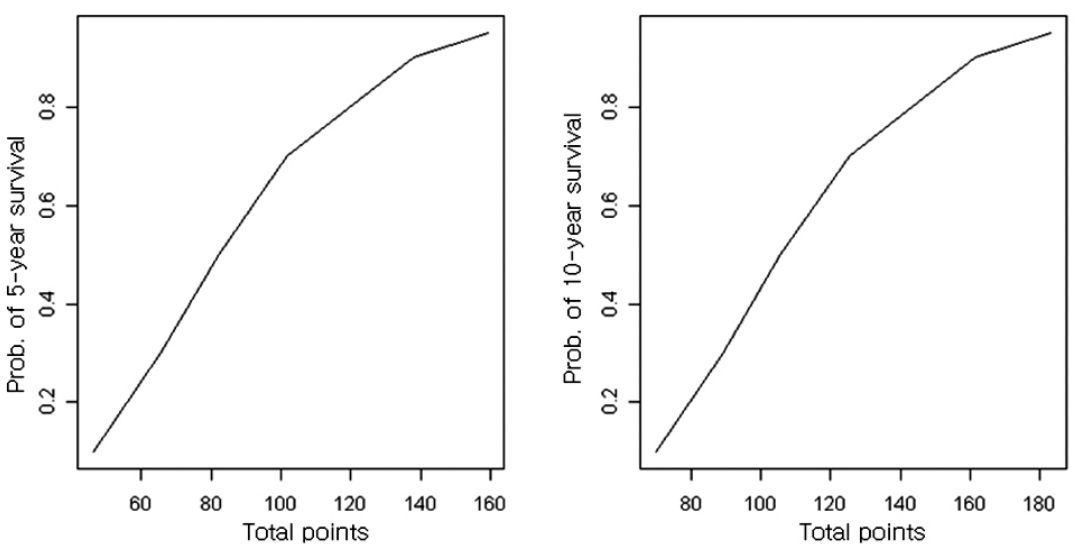

Fig. 2. The relationship between the total point derived from the nomogram and the probabilities of 5-year and 10-year survival.

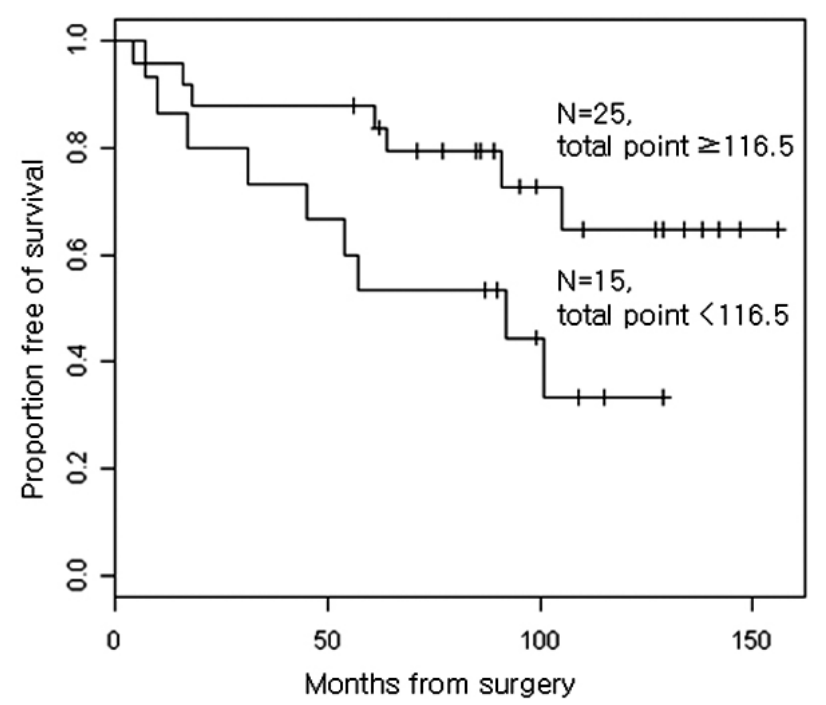

Fig. 3. Kaplan-Meier survival curves based on stratification using the total points assigned by the nomogram. The p-value comparing curves using Log rank test was less than 0.05 (Chi-square statistic was 3.9).

of independent prognostic factors, which provide an appreciation of the magnitude of impacts of individual factors on outcome probability (Kim et al., 2009). An accurate survival prediction model would be useful for patient counseling, planning follow-up, and selecting patients for additional treatment (Kim et al., 2004).

The data set that was used in this study had significantly different characteristics between early and advanced stages. In this case, identification of the predictable model for each stratified groups could be more reliable. In addition, no significant factors were detected in the advanced group when we performed multivariate analysis for each groups. Therefore, we developed and validated a nomogram for predicting 5-year and 10-year survival probability of OSCC patients in the early stage that underwent surgical resection. The nomogram predicts the probability of survival with a concordance index of 0.72 .

In this study, grade was found to be a key prognostic factor in the early stage group, while it was not significant in the advanced group. p53 was slightly significant in the early stage $(p=0.0857)$, which was previously shown not to be able to predict the progression of OSCC (Smith et al., 2009). However, we included p53 in the predictive model when constructing the nomogram, and explored the influence of p53. In this analysis, p53 was shown to be significant in the nomogram, and its high expression was correlated with the increased probability of survival even though it did not improve the c-index.

To evaluate the constructed nomogram, we determined whether the total points calculated by the nomogram could be used as a predictive factor, besides the c-index. The mean value of the total points was 116.5 , and we divided patients into two groups using this value. A new marker based on the total points was determined to be a significant factor in the survival model $(p<0.05)$. Therefore, a factor based on the total points can be a novel predictive marker. In addition, the randomization of patients in clinical trials by composite risk factors specific to OSCC would be possible using the nomogram.

This nomogram would be a useful tool for physicians who have to make decisions in the diagnosis of OSCC patients. For example, a patient with high expression of p53, G2 and T2 stage squamous cell carcinoma of the oral tongue is predicted to have a $79.8 \%$ and $60.7 \%$ probability of 5-year and 10-year survival, respectively. If the physicians can accurately estimate the probability of survival, they could be able to improve the life of patients by choosing more appropriate treatment.

In this study, we constructed a predictive nomogram for the early stage of OSCC, based on a sample size 
of 40 . This sample size may not be large enough to construct a predictive model. In addition, there might be more variables that we did not include in the model. However, this is the first prognostic nomogram developed to predict the survival of Korean patients. The constructed nomogram could be improved with more data and by including biological candidates in the predictive model.

\section{Acknowledgements}

This work was supported by Priority Research Centers Program through the National Research Foundation of Korea (NRF), funded by the Ministry of Education, Science and Technology (2010- 0029704).

\section{References}

Bianco, F.J.Jr. (2006). Nomograms and medicine. Eur. Urol. 50, 884-886.

Epstein, J.B., Sciubba, J., Silverman, S.Jr., and Sroussi, H.Y. (2007). Utility of toluidine blue in oral premalignant lesions and squamous cell carcinoma: continuing research and implications for clinical practice. Head Neck 29, 948-958.

Galli, P., Cadoni, G., Volante, M., De Feo, E., Amore, R., Giorgio, A., Arzani, D., Paludetti, G., Ricciardi, G., and Boccia, S. (2009). A case-control study on the combined effects of p53 and p73 polymorphisms on head and neck cancer risk in an Italian population. BMC Cancer 9 , 137.

Goulart, J.A., Nonaka, C.F., da Costa, M.C., de Almeida, R., and Galvao, H.C. (2009). Immunoexpression of cyclooxygenase-2 and p53 in oral squamous cell carcinoma. Am. J. Otolaryngol. 30, 89-94.

Hoang, T., Xu, R., Schiller, J.H., Bonomi, P., and Johnson, D.H. (2005). Clinical model to predict survival in chemonaive patients with advanced non-small-cell lung cancer treated with third-generation chemotherapy regimens based on eastern cooperative oncology group data. $J$. Clin. Oncol. 23, 175-183.

Karakiewicz, P.I., Briganti, A., Chun, F.K., Trinh, Q.D., Perrotte, P., Ficarra, V., Cindolo, L., De la Taille, A., Tostain, J., Mulders, P. F., Salomon, L., Zigeuner, R., Prayer-Galetti, T., Chautard, D., Valeri, A., Lechevallier,

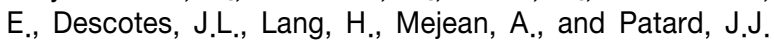
(2007). Multi-institutional validation of a new renal cancer-specific survival nomogram. J. Clin. Oncol. 25, 13161322.

Kattan, M.W., Eastham, J.A., Stapleton, A.M., Wheeler, T.M., and Scardino, P.T. (1998). A preoperative nomogram for disease recurrence following radical prostatectomy for prostate cancer. J. Natl. Cancer Inst. 90, 766771.

Kattan, M.W., Wheeler, T.M., and Scardino, P.T. (1999). Postoperative nomogram for disease recurrence after radical prostatectomy for prostate cancer. J. Clin. Oncol. 17, 1499-1507.

Kim, H.L., Seligson, D., Liu, X., Janzen, N., Bui, M.H., Yu, H., Shi, T., Figlin, R.A., Horvath, S., and Belldegrun, A.S. (2004). Using protein expressions to predict survival in clear cell renal carcinoma. Clin. Cancer Res. 10, 54645471.

Kim, M.S., Lee, S.Y., Lee, T.R., Cho, W.H., Song, W.S., Koh, J.S., Rouzier, R., Preti, M., Haddad, B., Martin, M., Micheletti, L., and Paniel, B.J. (2009). Prognostic nomogram for predicting the 5-year probability of developing metastasis after neo-adjuvant chemotherapy and definitive surgery for AJCC stage II extremity osteosarcoma. Ann. Oncol. 20, 955-960.

Myoung, H., Kim, M.J., Lee, J.H., Ok, Y.J., Paeng, J.Y., and Yun, P.Y. (2006). Correlation of proliferative markers (Ki-67 and PCNA) with survival and lymph node metastasis in oral squamous cell carcinoma: a clinical and histopathological analysis of 113 patients. Int. J. Oral Maxillofac. Surg. 35, 1005-1010.

Pisani, P., Parkin, D.M., Bray, F., and Ferlay, J. (1999). Estimates of the worldwide mortality from 25 cancers in 1990. Int. J. Cancer 83, 18-29.

Rouzier, R., Preti, M., Haddad, B., Martin, M., Micheletti, L., and Paniel, B.J. (2006). Development and validation of a nomogram for predicting outcome of patients with vulvar cancer. Obstet. Gynecol. 107, 672-677.

Rouzier, R., Pusztai, L., Delaloge, S., Gonzalez-Angulo, A.M., Andre, F., Hess, K.R., Buzdar, A.U., Garbay, J.R., Spielmann, M., Mathieu, M.C., Symmans, W.F., Wagner, P., Atallah, D., Valero, V., Berry, D.A., and Hortobagyi, G.N. (2005). Nomograms to predict pathologic complete response and metastasis-free survival after preoperative chemotherapy for breast cancer. J. Clin. Oncol. 23, 8331-8339.

Shah, N.G., Trivedi, T.I., Tankshali, R.A., Goswami, J.V., Jetly, D.H., Shukla, S.N., Shah, P.M., and Verma, R.J. (2009). Prognostic significance of molecular markers in oral squamous cell carcinoma: A multivariate analysis. Head Neck 31, 1544-1556.

Smith, J., Rattay, T., McConkey, C., Helliwell, T., and Mehanna, H. (2009). Biomarkers in dysplasia of the oral cavity: A systematic review. Oral Oncol, 45, 647-653.

Stephenson, A.J., Scardino, P.T., Eastham, J.A., Bianco, F.J.Jr., Dotan, Z.A., DiBlasio, C.J., Reuther, A., Klein, E.A., and Kattan, M.W. (2005a). Postoperative nomogram predicting the 10-year probability of prostate cancer recurrence after radical prostatectomy. J. Clin. Oncol. 23, 7005-7012.

Stephenson, A.J., Smith, A., Kattan, M.W., Satagopan, J., Reuter, V.E., Scardino, P.T., and Gerald, W.L. (2005b). Integration of gene expression profiling and clinical variables to predict prostate carcinoma recurrence after radical prostatectomy. Cancer 104, 290-298.

Sudbo, J., Reith, A. (2005). The evolution of predictive oncology and molecular-based therapy for oral cancer prevention. Int. J. Cancer 115, 339-345. 